\title{
¿Qué sucede cuando los historiadores pierden su propia historia? Aporte a la discusión sobre el perfil histórico de la Asociación de Historiadores Latinoamericanistas Europeos
}

\author{
What happen when the historians lose their own history? Contribution \\ to the dialog on the Asociación de Historiadores Latinoamericanistas \\ Europeos's Profile
}

GIRAUDO, Laura (coord.). Historia de AHILA: perfil de la Asociación de Historiadores Latinoamericanistas Europeos (1969-2008). Colección Estudios de Historia Latinoamericana. Madrid; Frankfurt: Iberoamericana; Vervuert, 2008, 210 p.

\section{duque.cesar@ur.edu.co \\ Estudiante de pregrado \\ Universidad del Rosario \\ C20 Calle 55 n 77 B-24/103 \\ Bogotá \\ Colombia}

César Augusto Duque Sánchez

Palabras clave

Historiador; América Latina; Observación histórica.

Keywords

Historian; Latin America; Historical note. 
La profesora Laura Giraudo publicó en el año 2008 un texto sobre la Asociación de Historiadores Latinoamericanistas Europeos (AHILA) que concluyó una etapa de investigación en los archivos privados de los miembros fundadores de la asociación.

La investigación exigió la revisión de todas las actas de las reuniones de la institución; el análisis de publicaciones como Historia Latinoamericana en Europa (1986-2002) e Historia Latinoamericana en Europa y América (2003-2007); cinco entrevistas con miembros consagrados de la asociación; y el intercambio epistolar entre Laura Giraudo, Horst Piechmann y Ádám Anderle.

El libro está dividido en tres partes. La primera, "Historia de AHILA: de las ideas a la organización", corresponde a la "historia de AHILA" escrita por la profesora Giraudo. La segunda parte, "Palabras y documentos para una historia de AHILA", contiene una entrevista hecha por Giraudo a Francisco Morales Padrón, cuatro artículos alusivos a la historia de AHILA (dos de Magnus Mörner, uno de Ádám Anderle, uno de John Fisher) y un compendio con las actas de la asociación.

Laura Giraudo decidió llamar a la investigación "Perfil de la Asociación de Historiadores Latinoamericanistas Europeos". Esta es la ocasión para celebrar la escritura de un perfil - no una crónica, no un ensayo, no un diálogo, no unas memorias, sino un perfil - sobre lo que, en términos generales, podría definirse como una "agremiación" de científicos sociales.

Un perfil describe el objeto que ha escogido como materia de observación y reconoce en él los rasgos de continuidad que lo caracterizan en el tiempo. Este perfil, como cualquier otro, tiene un objetivo divulgativo y uno estratégico.

El primer objetivo es presentar la Asociación de Historiadores Latinoamericanistas Europeos a un público amplio de historiadores que desconocen la trayectoria de la institución. El segundo objetivo es examinar la identidad de AHILA a la luz de sus treinta y nueve años de trayectoria.

Giraudo escribió una "historia" descriptiva - no analítica, no crítica, no problema - y aunque ese formato de lectura es muy distinto al que acostumbran a leer los historiadores "académicos" en la actualidad, bastará con señalar los principales "nudos" argumentativos del texto toda vez que eluciden su problemática central.

El perfil de AHILA recoge implícitamente varias dudas internas que se plantearon los miembros de la asociación por lo menos diez años antes de 2008.

Este libro posee las características de un recurso de agnición, no las de una investigación sobre un caso particular capaz de elucidar el problema general de los retos y los desafíos de los historiadores europeos. El libro es la "ruta de navegación" de la agremiación tras las peripecias gremiales sufridas en la Postguerra Fría.

La obra debe leerse en el orden de tres secciones que revelan preguntas nucleares para los miembros de la institución. Esas secciones son:

A. "Genealógica". En ella, Giraudo busca las pautas que dieron origen a la identidad europea de AHILA.

B. "Gnoseológica". En ella, Giraudo enuncia la finalidad institucional 
de AHILA frente al conocimiento histórico.

C. "Práctica". En ella, Giraudo incluye las peripecias institucionales del decenio de los años 1980, que produjo una pérdida de rumbo al interior de la institución y la resolución - en la práctica - de esa confusión.

\section{Sección "genealógica"}

El primer bloque de inquietudes se resume en la pregunta: ¿cuál es el origen de la identidad europea de AHILA? Esa inquietud se recoge en los capítulos 1 , 2,3 y 5 .

En un inicio (entre 1969 y 1978), los argumentos de los miembros de AHILA para justificar la existencia de una agremiación europea interesada en estudios de historia latinoamericana derivaron de agudas reflexiones sobre la carencia de condiciones tecnológicas, administrativas y económicas propicias para la concurrencia de miembros no europeos a las reuniones de la institución.

En su primer periodo, AHILA fue una institución de escasos recursos que no podía atender al interés de ampliar el rango de convocatoria $y$, por eso, se constituyó como una organización de historiadores europeos, residentes en Europa y dedicados a América Latina.

El periodo de AHILA como institución europea cubrió una porción importante de su historia institucional. Sin embargo, el tema de la identidad europea de la asociación no fue resuelto verbalmente una vez que desaparecieron las limitaciones tecnológicas, económicas y políticas que impedían la apertura 262 mundial de la asociación.

Los capítulos 4, 6 y, parcialmente, el 7 se preguntan: ¿qué hizo AHILA para contribuir a la construcción de un escenario de discusión transregional? y ¿cómo transformó las barreras nacionales que separaban la comunicación entre historiadores europeos?

Las estrategias institucionales para flexibilizar los diálogos entre historiadores en el plano internacional y el interés por crear plataformas organizativas y de discusión crearon espacios académicos convocantes en torno al diálogo científico (MÖRNER 2008, p. 109-123).

Magnus Mörner indicaba - en un documento adjunto en el libro de Giraudo - que la creación de esas plataformas había permitido la colaboración internacional entre historiadores con resultados muy positivos que beneficiaron la producción de un conocimiento europeo sobre la historia latinoamericana (MÖRNER 2008, p. 109-123).

Además, esas plataformas también contribuyeron a la superación del aislamiento académico en el que se encontraban los historiadores latinoamericanistas de algunos países europeos, quienes se unieron a la promoción del uso generalizado de los idiomas latinoamericanos - español y portugués - entre los miembros de la asociación (MÖRNER 2008, p. 109-123).

Tomados con rigurosidad, y con vistas a investigar sobre un marco más general, los documentos consultados en este bloque serían de gran utilidad para la historia del "campo académico" de las ciencias sociales europeas durante la Guerra Fría. 
La lectura sugiere, además, un mapa institucional de las asociaciones que componen ese campo. En dicho mapa son mencionadas las relaciones que sostiene AHILA - institución continental - con las instituciones nacionales y mundiales dedicadas a financiar o promover el conocimiento histórico.

\section{Sección "gnoseológica"}

El tercer bloque, que, a mi juicio, es el más importante para los propósitos estratégicos de la obra, evalúa el trabajo académico de la Asociación y aporta un rasgo científico a la caracterización del trabajo hecho por AHILA. La pregunta que secunda este bloque es: ¿Qué contribuciones ha hecho AHILA al conocimiento histórico de América Latina y por qué medios?

Este segmento recoge los contenidos del capítulo 7 y, parcialmente, los del capítulo 8 del libro. El eje de esta sección es el proceso de creación y consolidación de una comunidad científica con "independencia".

Los boletines informativos y los cuadernos de investigación que ofrecían mayor cobertura informativa para los miembros daban reportes de investigación al público interesado por los avances de la asociación en materia de conocimiento. Esos boletines, mirados en perspectiva, fueron una fuente que aportó mucho material para la investigación.

Tomando esos capítulos como base, el lector puede estudiar los factores que flexibilizaron las temáticas de los congresos de AHILA. Al mismo tiempo, puede evaluar las consecuencias que tuvieron esas temáticas generales en el contenido de la historiografía producida por los historiadores latinoamericanistas europeos.

De allí se puede concluir que los congresos y los encuentros académicos fueron decisivos para la organización de una agenda de investigación e incentivaron grupos de investigación por temáticas afines.

A mi juicio, este bloque - interesado en los aportes de AHILA a la historiografía latinoamericana y al corpus social interesado en Latinoamérica es el único suelo que debería permanecer fijo como factor de continuidad para elaborar las rutas de la institución.

En él, sus miembros - los de AHILA - reconocerán los horizontes perdidos de una sociabilidad histórica, una sociedad de conocimiento que actualmente posee el núcleo fuerte de una ecúmene transregional de historia latinoamericana.

\section{Sección "práctica"}

Sobre la última sección, dedicada a las conclusiones, queda agregar que los decenios de 1980 y 1990 impusieron las siguientes características a la institución: AHILA estuvo confinada al cultivo de una nueva generación de latinoamericanistas.

De este bloque se concluye que la institución mejoró el nivel de cooperación y el desarrollo de una historiografía sin un referente territorial inmediato; y que presenció la eliminación de las fronteras ideológicas en el medio de la Cortina de Hierro.

El texto hace justicia al reconocer que el incremento de miembros asociados trajo consigo: la creación de grupos de trabajo; una mayor organización interna; 
un aumento en la frecuencia de las reuniones del Comité Central (Directivo); la elección de temas generales para los congresos trienales; las variaciones en la identidad de la Revista; y, por supuesto, un aumento de fondos.

Esos son puntos de partida a favor de la investigación escrita por Laura Giraudo, quien enunció puntos de alta relevancia para concretizar la investigación historiográfica en un escenario social. A partir de esos puntos, la investigación historiográfica se enfrenta a una invitación sugestiva que perfila una transición de la historiografía formal o intelectual a la historia social de la historiografía en América Latina.

Por lo demás, queda sostener lo ya dicho: que las prospectivas jamás se podrán elaborar sin un buen diario de navegación y, cuando algunos historiadores escriben su propia historia, pierden de vista que la historia como profesión es una herramienta para saber conducir la identidad.

Es importante reconocer que ese no es el caso de Giraudo, sino de otros intentos efusivos de historiar a AHILA y que se incluyen en las páginas finales, como archivos anexos, del perfil que hoy se reseña.

La historia es una ciencia en la que son evaluados por igual los esfuerzos y sus resultados, siempre que los efectos investigativos sean producto de la sumatoria de sus causas.

Por eso y todo lo dicho, se puede concluir que el texto de Giraudo se aceleró en su proceso de publicación, pues está relacionado en un orden sucesivo inconexo; que los debates que lo anticiparon requieren un punto de referencia

264 más claro para promover la discusión; que es necesario echar un nuevo vistazo al perfil de la institución.

Con esta reseña, espero haber contribuido en algo a organizar el trabajo que con esmero realizó la profesora; su perfil es - repito - digno de celebrarse porque revive una vez más la historia como género estratégico para ayudar a definir el rumbo de la vida en sociedad.

\section{Referencias bibliográficas}

MÖRNER, Magnus. AHILA y la comunidad internacional de historiadores latinoamericanistas: un breve informe sobre el periodo 1970-1990. In: GIRAUDO, Laura (coord.). Historia de AHILA: perfil de la Asociación de Historiadores Latinoamericanistas Europeos (1969-2008). Colección Estudios de Historia Latinoamericana. Madrid; Frankfurt: Iberoamericana; Vervuert, 2008, p. 109-120.

GIRAUDO, Laura (coord.). Historia de AHILA: perfil de la Asociación de Historiadores Latinoamericanistas Europeos (1969-2008). Colección Estudios de Historia Latinoamericana. Madrid; Frankfurt: Iberoamericana; Vervuert, 2008. 